\title{
Analgesia epidural com xilazina ou detomidina em cães submetidos a procedimentos ortopédicos
}

\section{Epidural analgesia with xylazine or detomidine in dogs undergoing orthopedic procedures}

\section{Resumo}

Os agonistas dos $\alpha 2$-adrenorreceptores podem ser classificados como sedativo-hipnóticos e têm propriedades adicionais miorrelaxantes e analgésicas, portanto, por apresentarem tais características, eles são alternativas ao uso dos opioides, via epidural. Assim, foram avaliados em catorze cães os efeitos analgésicos e cardiorrespiratórios causados pela injeção epidural de xilazina ou detomidina, para comparar o grau de analgesia proporcionado por ambos. Utilizou-se a escala de Melbourne, considerando como critérios para a administração da analgesia de resgate uma pontuação de 13 pontos. Os grupos não apresentaram diferenças significativas e não necessitaram de resgate analgésico. A conclusão foi que os dois fármacos produzem analgesia intensa durante os períodos transcirúrgico e pós-operatório sem causar qualquer depressão respiratória, bradicardia ou hipotensão.

\section{Abstract}

The agonists of $\alpha 2$-adrenoceptors may be classified as a sedativehypnotic and have additional myorelaxant and analgesic properties, therefore, for presenting such characteristics, they are alternatives to using opioids, via epidural. Thus, it was evaluated in fourteen dogs the analgesic and cardiorespiratory effects caused by epidural injection of xylazine or detomidine, to compare the level of analgesia provided by both products. The range of Melbourne was applied for performing the comparison, considering as criteria for the administration of analgesia rescue a score of 13 points. Both groups did not present any significant changes and not required analgesic rescue. The conclusion was that the two drugs produce intense analgesia during the transurgical and postoperative periods without any effect on respiratory depression, bradycardia or hypotension. 
Maria Olivo de Cerqueira ${ }^{1}$

Celso Braga Sobrinho²

Dayane de Oliveira Ferreira ${ }^{3}$

Jordana Casemiro Pinto Monteiro ${ }^{4}$
Rua Bragança, 404, Parque Enseada

Guarujá/SP, CEP: 11443-080

ه mah_2201@hotmail.com

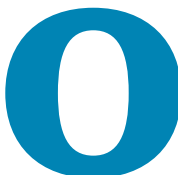

s agonistas dos a2-adrenorreceptores, classificados como sedativo-hipnóticos e com propriedades adicionais miorrelaxantes e analgésicas (POHL, 2010), são amplamente utilizados para contenção química e medicação pré-anestésica em pequenos e grandes animais. Tais propriedades mediadas nos receptores $\alpha 2$-centrais têm como mecanismo primário a diminuição na liberação da noradrenalina e, portanto, inibição da transmissão do impulso. A sedação é atribuída à depressão dos neurônios de uma região do tronco encefálico na qual os impulsos são transmitidos ao prosencéfalo e ao sistema límbico (BRAGA, 2012). As vantagens da anestesia epidural sobre a anestesia geral incluem: a segurança, a minimização das alterações cardiorrespiratórias, a facilidade de execução da técnica e a acessibilidade de seu custo (MASSONE, 2011).

\section{Keywords}

Agonists of $\alpha 2$-adrenoceptors. Epidural. Analgesia.

1 Médica-veterinária graduada pela Universidade Metodista de São Paulo/SP, Brasil.

2 Docente no curso de Medicina Veterinária na Universidade Metodista e na Universidade Anhanguera, São Paulo/SP, Brasil.

3 Médica-veterinária graduada pela Universidade Metodista de São Paulo e responsável pelo setor de anestesia no Hospital Veterinário Metodista. Sócia proprietária da clínica veterinária Puppy Brasil.

${ }^{4}$ Médica-veterinária graduada pela Universidade Metodista de São Paulo. Anestesista pelo setor de anestesiologia do Provet São Paulo. 


\section{Revisão de literatura}

A presença de dor no período pós-operatório imediato gera efeitos adversos na qualidade de vida do paciente (MARQUES; PEREIRA; MARQUES, 2009). A anestesia epidural desperta grande interesse por seu baixo custo, facilidade de execução, bom relaxamento muscular, mínima depressão dos sistemas orgânicos e rápida recuperação. Esta técnica é efetiva no tratamento da dor aguda e crônica, assim como na analgesia pré-operatória, transoperatória e pós-operatória, tanto nos seres humanos como em animais (LAVOR et al., 2004). O uso epidural dos agonistas $\alpha 2$ foi bem descrito na literatura em diversas espécies (ALMEIDA et al., 2004). Desde a sua introdução no século passado, a xilazina tem sido utilizada na composição da anestesia balanceada, por meio de agentes farmacológicos, em várias espécies animais (BRICEÑO et al., 2012).

Mataqueiro et al. (2000) relataram o efeito antinociceptivo de todos os a2-agonistas, mas utilizaram doses que também foram capazes de causar outros efeitos, como sedação, relaxamento muscular e depressão respiratória. O uso conjunto de agonistas dos receptores $\alpha 2$ e opioides pode ser de grande utilidade, já que as duas substâncias têm sítios de ligação específicos na medula espinhal (VALADÃO; DUQUE; FARIAS, 2002). Rosa (2014) relatou que a detomidina possui característica altamente lipofílica e é rapidamente absorvido via intramuscular, possuindo grande afinidade pelo sistema nervoso central, sendo considerado cinquenta vezes mais potente que a xilazina. Ribeiro et al. (2012) observaram que, assim como com a xilazina, a profundidade e a duração da sedação e da analgesia promovidas pela detomidina são dose-dependentes.

Cirurgias ortopédicas de membros inferiores causam dor pós-cirúrgica de grau moderado a severo e justificam a utilização de fármacos associados ou não a opioides por via epidural. Entretanto, o conhecimento desses anestésicos pode ser importante para comparação da analgesia por eles produzida e observação da ocorrência de possíveis efeitos colaterais. A lidocaína é um fármaco de curta ação com utilização limitada em cirurgias prolongadas e, quando empregada em tais situações, há necessidade da sua associação com fármacos de longa duração (GASPARINI et al., 2007). De tal forma, a lidocaína em associação com a xilazina produz a extensão cranial do bloqueio, atingindo a região entre a última vértebra torácica e a primeira lombar (GASPARINI et al., 2007), o que possibilita a realização de procedimentos ortopédicos.

Lavor et al. (2004), avaliando os efeitos do emprego da lidocaína isolada, não associada a outros fármacos, constataram apenas o efeito anestésico caudal, já os fármacos xilazina e cetamina produziram analgesia segmentar, bem como certo grau de depressão do sistema nervoso central com analgesia geral e sedação. A xilazina é um fármaco pré-anestésico a2-agonista usado frequentemente na medicina veterinária, com efeito similar quando administrada via endovenosa ou intramuscular (PRADO FILHO et al.,2000). A administração epidural de xilazina possui vantagens em relação à forma sistêmica (POHL, 2010). Em cães anestesiados com isoflurano, a xilazina administrada via epidural produz analgesia por um período superior a quatro horas e os efeitos cardiorrespiratórios observados são mínimos (POHL, 2010).

Brondani et al. (2004) destacaram que a administração de agonistas $\alpha 2$-adrenérgicos via epidural é uma alternativa analgésica aos opioides, possuindo como vantagens as ausências de prurido, êmese e desenvolvimento de tolerância e dependência. Esses autores também analisaram o emprego da analgesia epidural efetuada com clonidina ou romifidina em cães submetidos à cirurgia coxofemoral e concluíram que os dois fármacos determinaram intensa analgesia transcirúrgica sem depressão respiratória e analgesia pós-operatória pouco intensa, por duas horas. Porém, quando o fármaco romifidina foi empregado ocorreram alterações fisiológicas. A analgesia produzida pela administração de agonistas $\alpha 2$-adrenérgicos foi comprovada em diversas espécies. Em cães, a administração epidural de xilazina produz analgesia por um período superior a quatro horas, com efeitos cardiorrespiratórios mínimos (POHL, 2010).

Silva (2009) analisou a analgesia via peridural de lidocaína associada à xilazina ou detomidina na prevenção da dor pós-incisional em éguas e os resultados encontrados demonstraram que a lidocaína reduziu a hiperalgesia pós-incisional. Entretanto, as associações de lidocaína com os agonistas $\alpha 2$, xilazina e detomidina proporcionaram redução ainda mais significativa da hiperalgesia pós-incisional, interrompendo ou diminuindo os mecanismos que processam a informação nociceptiva espinhal e supraespinhal, com administração de doses baixas desses fármacos via peridural.

Braga (2012) verificou que cães e gatos tratados com xilazina e medetomidina apresentaram bradicardia, com redução na frequência cardíaca em até $60 \%$, iniciando-se 10 minutos após a administração e podendo durar por até 60 minutos. A xilazina apresenta maiores efeitos no sistema cardiovascular quando em associação com a cetamina; a diminuição da fração de encurtamento da fibra miocárdica se deve ao efeito depressor da xilazina (CARVALHO, 2007).

A detomidina, quando comparada à xilazina, apresenta efeitos analgésicos, sedativos e hipnóticos mais pronunciados e com maior durabilidade, tornando-se segura para procedimentos cirúrgicos, diagnósticos e terapêuticos na espécie equina (BRAGA, 2012). Em equinos, a xilazina apresentou menor redução na frequência cardíaca, quando comparada com detomidina e romifidina em doses equipotentes (BRAGA, 2012). 
Em cães, Pohl (2010) observou que, quando é empregada a detomidina via epidural, os animais apresentaram valores mais elevados de pressões arteriais e significativa hipertensão após a indução da anestesia epidural. Já quando foi utilizada a dexmedetomidina, foi constatada uma acentuada ação analgésica quando o produto foi empregado via epidural, apresentando efeito dose-dependente superior ao obtido pela administração intravenosa e o seu efeito foi correlacionado à sua intensa afinidade por receptores a2-adrenérgicos situados na medula espinhal.

A anestesia epidural desperta grande interesse por seu baixo custo, facilidade de execução, obtenção de bom relaxamento muscular, mínima depressão dos sistemas orgânicos e rápida recuperação. Essa técnica é efetiva no tratamento da dor aguda e crônica, assim como nas analgesias pré-operatória, transoperatória e pós-operatória, tanto nos seres humanos como em animais (LAVOR et al., 2004).

\section{Material e métodos}

O trabalho foi aprovado (Protocolo $\left.\mathrm{n}^{\circ} 130 / 2014\right)$ pela Comissão de Ética no Uso de Animais (Ceua) da Escola de Ciências Médicas e da Saúde da Universidade Metodista de São Paulo. Foram utilizados 14 cães adultos, de sexo, raça e peso variados, provenientes do Hospital Veterinário da Universidade Metodista de São Paulo (Umesp), classificados como paciente saudável (ASA I) e paciente com doença sistêmica leve ou moderada, sem limitação funcional (ASA II), submetidos a procedimentos ortopédicos nos membros pélvicos. Os animais foram submetidos a jejum alimentar de oito horas, que antecederam à anestesia e ao procedimento cirúrgico.

Os cães foram distribuídos aleatoriamente em dois grupos de sete animais, xilazina (grupo 1) e detomidina (grupo 2). Todos os animais receberam a medicação pré-anestésica constituída pela associação de acepromazina e petidina nas doses de 0,05 e $5 \mathrm{mg} / \mathrm{kg}$, respectivamente, via intramuscular. Decorridos 20 minutos da pré-anestesia, foi realizada a indução anestésica com propofol $(5 \mathrm{mg} / \mathrm{kg})$ por injeção intravenosa, visando a rápida elevação da sua concentração no sangue para um nível eficaz (bolus). Após a indução anestésica, foi administrada via epidural a xilazina na dose de $0,25 \mathrm{mg} / \mathrm{kg}$ aos animais do grupo 1 ou detomidina na dose de $30 \mu \mathrm{g} / \mathrm{kg}$ nos animais do grupo 2, diluídos em solução salina $0,9 \%$ perfazendo um volume final de $0,3 \mathrm{ml} /$ $\mathrm{kg}$. Os animais foram intubados e mantidos sob anestesia geral inalatória com isoflurano a uma concentração de $2 \%$ no ar inspirado.

A frequência cardíaca $(\mathrm{FC})$ e a respiratória $(f)$ e a pressão arterial sistólica (PAS) foram avaliadas 10 minutos antes da indução anestésica com auscultação manual e doppler, e a cada 10 minutos após punção epidural; a frequência cardíaca se baseou na do monitor anestésico, a respiratória de acordo com as movimentações do balão e a pressão arterial sistólica mensurada com doppler até o final do procedimento cirúrgico. Durante o período transoperatório e na próxima hora de pós-operatório imediato os animais foram mantidos em colchão térmico e a temperatura retal acompanhada e mantida na faixa de variação fisiológica para cães. Após o término da cirurgia, o grau de analgesia foi avaliado com o emprego dos escores da escala de Melbourne (Tabela 1), com intervalos de 30 minutos durante uma hora.

Este trabalho considerou escore a partir de 13 como valor absoluto para a utilização de analgesia de resgate, administrando-se, quando necessário, tramadol na dose de $2 \mathrm{mg} / \mathrm{kg}$ intramuscular para controle da dor pós-cirúrgica. Os escores mais baixos indicam melhor analgesia, enquanto os mais altos demonstram a necessidade de analgesia de resgate, seguindo o padrão de avaliação analgésica encontrado na literatura. Os resultados obtidos foram analisados com o emprego do programa computacional Statistical Analysis System (SAS). A normalidade dos resíduos foi avaliada pelo teste de Shapiro-Wilk (proc univariate) e a homogeneidade das variâncias comparadas pelo teste qui-quadrado (comando spec do proc glm). Os dados foram submetidos à análise de variância (proc glm), que considerou o tipo de anestésico utilizado e o tempo sobre escore, $\mathrm{FC}, f$, PAS e To ${ }^{\circ}$, sendo que, em seguida, foi utilizado o teste de comparação de médias Tukey. O nível de significância adotado foi o de $5 \%$.

\section{Resultados}

Os animais de ambos os grupos não apresentaram mudanças significativas (Tabela 2). No período pós-operatório, o escore (Figura 1) se manteve dentro da faixa de variação, não necessitando de resgate analgésico, porém foi observada tendência estatística na analgesia entre os grupos. Em contrapartida, o escore em equinos anestesiados por via epidural possui uma duração de até duas horas e meia em ambos, porém em cães a xilazina (XIL) pode persistir por até quatro horas.

Durante a observação do eletrocardiograma, um animal do grupo detomidina (DET) apresentou arritmias como bloqueio átrio ventricular de $2^{\circ}$ nos primeiros 30 minutos após a administração epidural do fármaco e considerável diminuição da FC em dois animais no grupo XIL, que foram prontamente tratadas com aplicação de atropina $(0,03 \mathrm{mg} / \mathrm{kg})$ intravenoso, porém estatisticamente os animais se mantiveram dentro da faixa de variação (Figura 2). Ambos os grupos não apresentaram valores alterados de pressões arteriais (Figura 3). Os valores de $f$ não diferiram do controle em nenhum dos grupos tratados em momento algum avaliado (Figura 4 ). 


\begin{tabular}{|c|c|c|}
\hline OBSERVAÇÃO & SCORE & CARACTERISTICAS \\
\hline \multirow{3}{*}{ FC } & 1 & $>20 \%$ valor basal \\
\hline & 2 & $>50 \%$ valor basal \\
\hline & 3 & $>100 \%$ valor basal \\
\hline \multirow{3}{*}{$f$} & 1 & $>20 \%$ valor basal \\
\hline & 2 & $>50 \%$ valor basal \\
\hline & 3 & $>100 \%$ valor basal \\
\hline \multirow{3}{*}{ PAS } & 1 & $>20 \%$ valor basal \\
\hline & 2 & $>50 \%$ valor basal \\
\hline & 3 & $>100 \%$ valor basal \\
\hline Temperatura retal & 1 & (Acima do valor basal) \\
\hline Salivação & 2 & \\
\hline Pupilas dilatadas & 2 & \\
\hline \multirow{3}{*}{ Resposta à palpação } & 0 & Normal \\
\hline & 2 & Reage/protege a ferida no momento do toque \\
\hline & 3 & Reage/protege a ferida antes do toque \\
\hline \multirow{6}{*}{ Atividade } & 0 & Dormindo \\
\hline & 0 & Semiconsciente \\
\hline & 1 & Acordado \\
\hline & 0 & Alimenta-se \\
\hline & 2 & Agitado \\
\hline & 3 & Mudanças contínuas de posição, mutilação \\
\hline \multirow{4}{*}{ Status mental } & 0 & Dócil \\
\hline & 1 & Amigável \\
\hline & 2 & Cauteloso \\
\hline & 3 & Alerta \\
\hline \multirow[t]{7}{*}{ Postura } & 2 & Protege a área afetada \\
\hline & 0 & Decúbito lateral \\
\hline & 1 & Decúbito esternal \\
\hline & 1 & Sentado ou em pé, cabeça elevada \\
\hline & 2 & Em pé, cabeça baixa \\
\hline & 1 & Movimenta-se \\
\hline & 2 & Postura anormal \\
\hline \multirow{4}{*}{ Vocalização } & 0 & Não vocaliza \\
\hline & 1 & Vocaliza quando tocado \\
\hline & 2 & Vocalização intermitente \\
\hline & 3 & Vocalização contínua \\
\hline
\end{tabular}

Tabela 1 - Escala de Dor da Universidade de Melbourne. Fonte: Firth e Haldane (1999), retirado de Pohl (2010).

\section{GRUPO}

10 MINUTOS

20 MINUTOS

30 MINUTOS

\begin{tabular}{|c|c|c|c|}
\hline \multicolumn{4}{|c|}{ FC } \\
\hline XIL & $83 \pm 24^{A}$ & $96 \pm 22^{A}$ & $89 \pm 13^{A}$ \\
\hline DET & $88 \pm 26^{A}$ & $102 \pm 19^{A}$ & $98 \pm 15^{A}$ \\
\hline \multicolumn{4}{|c|}{$f$} \\
\hline XIL & $10 \pm 4^{\mathrm{A}}$ & $10 \pm 2^{A}$ & $8 \pm 4^{A}$ \\
\hline DET & $16 \pm 4^{\mathrm{B}}$ & $16 \pm 6^{8}$ & $14 \pm 6^{A B}$ \\
\hline \multicolumn{4}{|c|}{ PAS } \\
\hline XIL & $96 \pm 41^{A}$ & $97 \pm 30^{A}$ & $101 \pm 29^{A}$ \\
\hline DET & $105 \pm 34^{A}$ & $115 \pm 41^{A}$ & $105 \pm 40^{A}$ \\
\hline \multicolumn{4}{|c|}{$\mathrm{T}^{\circ}$} \\
\hline XIL & $37 \pm 0^{A}$ & $36 \pm 1^{A}$ & $36 \pm 1^{A}$ \\
\hline DET & $37 \pm 0^{A}$ & $36 \pm 1^{A}$ & $36 \pm 1^{A}$ \\
\hline
\end{tabular}

Tabela 2 - Valores transoperatórios de FC, $f$, PAS, To, obtidos em cães submetidos a cirurgias ortopédicas com xilazina (XIL) ou detomidina (DET) aplicadas pela via epidural. Fonte: Arquivo pessoal. Letras diferentes indicam diferença estatística entre grupos. Valores expressos em média \pm desvio padrão.

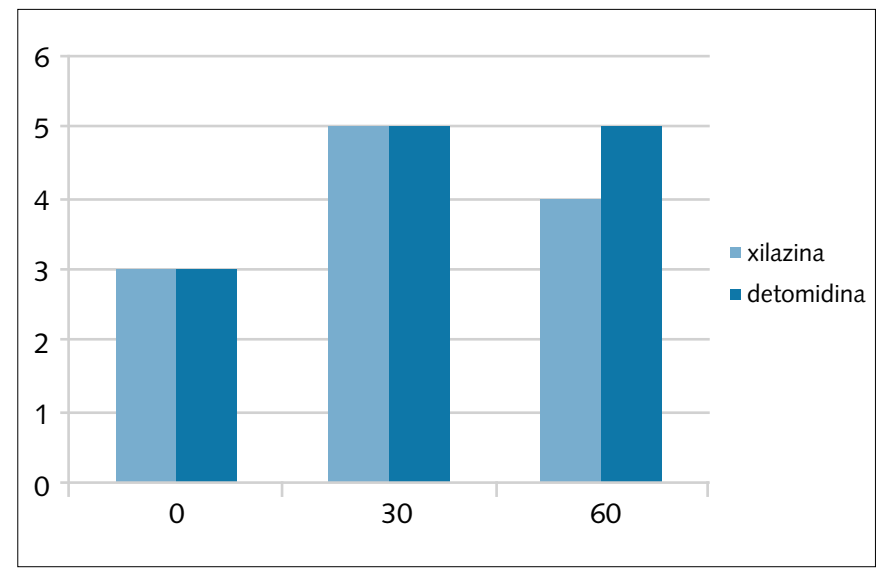

Figura 1 - Médias do escore no período pós-operatório.

Fonte: Arquivo pessoal.

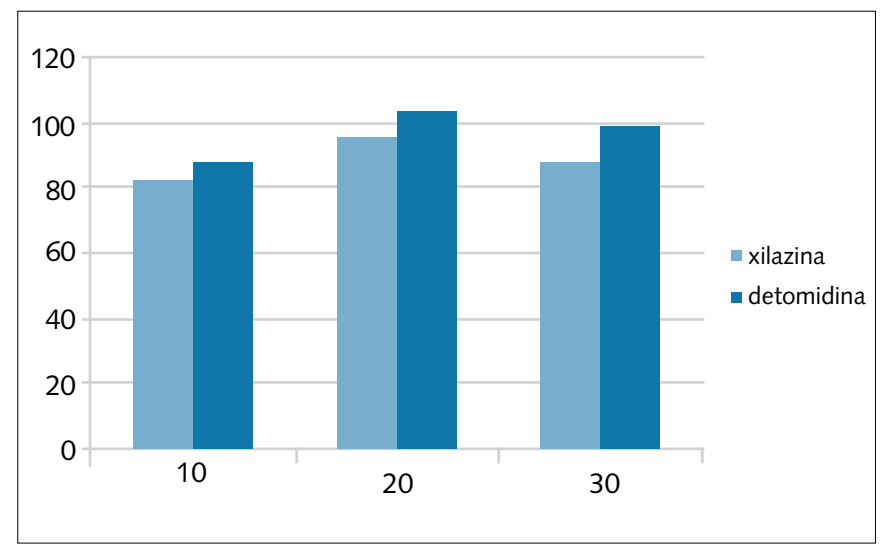

Figura 2 - Média da frequência cardíaca no período transoperatório. Fonte: Arquivo pessoal. 


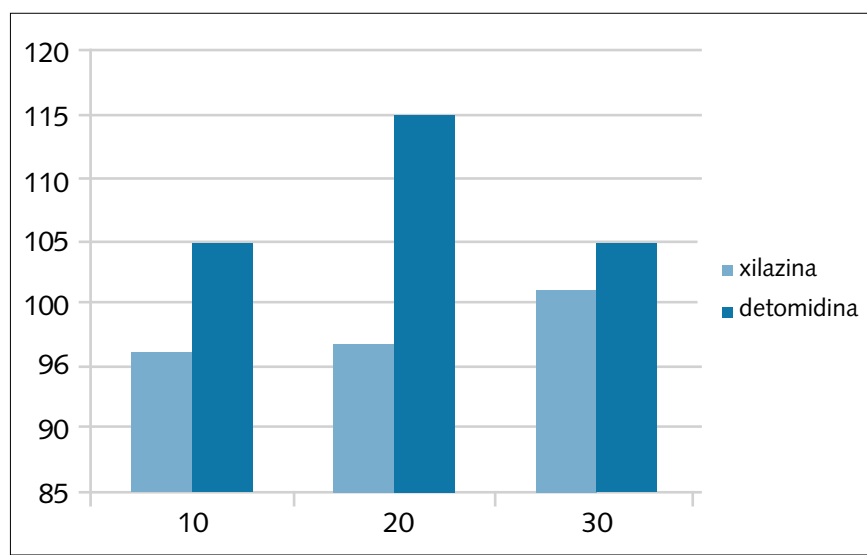

Figura 3 - Média da pressão arterial sistólica no período transoperatório. Fonte: Arquivo pessoal.

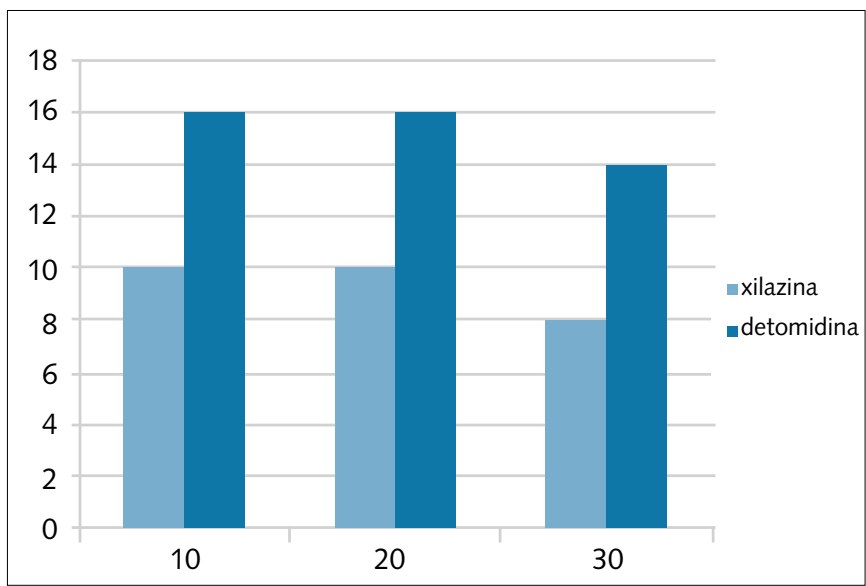

Figura 4 - Média da frequência respiratória no período transoperatório. Fonte: Arquivo pessoal.

\section{Discussão}

Neste trabalho não foi observado aumento das pressões arteriais em nenhum dos grupos tratados, após a administração epidural de xilazina ou detomidina. De fato, Pohl (2010) verificou que esse período inicial de vasoconstrição e hipertensão é originado pela estimulação de receptores a2 e a1 vasculares, sendo parcialmente responsável pelo desenvolvimento de bradiarritmias, devido a aumentos na atividade barorreflexa e no tônus vagal.

Marques, Pereira e Marques (2009) verificaram que os fármacos a2-agonistas causam bradicardia, comumente acompanhada por bloqueio atrioventricular, bloqueio sinoatrial menos frequente e redução no débito cardíaco, considerando-se que, na maioria dos animais, os bloqueios são mais intensos nos primeiros minutos após a administração. Após esse período, a frequência cardíaca eleva-se gradativamente e os bloqueios desaparecem. A bradicardia e os bloqueios cardíacos persistem por mais tempo após a administração de romifidina do que com xilazina ou detomidina. Em cães, a administração epidural de xilazina não induz alterações significativas na pressão arterial (POHL, 2010), sendo observado resultado semelhante neste estudo, o qual foi igual ao controle em todos os momentos avaliados.

Ribeiro et al. (2012) ressaltam que a xilazina, via intravenosa e administrada em bovinos, ao mesmo tempo que promove uma sedação mais intensa e prolongada que a detomidina, induz a uma maior quantidade de efeitos indesejáveis, como salivação, decúbito, redução das frequências cardíaca e respiratória, pressão arterial média, motilidade ruminal e temperatura, sendo essas alterações, da mesma forma, mais prolongadas. Guirro et al. (2009) relatam que a xilazina é o agonista $\alpha 2$ mais utilizado em grandes animais e, quando administrada via epidural, produz analgesia perineal sem alterar as variáveis cardiorrespiratórias em equinos. Assim como observado neste trabalho, não obtivemos alterações cardiorrespiratórias significantes e nem sinais clínicos indesejáveis.

Entre os fármacos utilizados neste estudo, a detomidina induziu hipertensão mais intensa, corroborando dados que indicam que a sua administração epidural em bovinos produz hipertensão moderada (POHL, 2010). Brondani et al. (2004) destacaram que a ação dos agonistas a 2 sobre a pressão arterial é bastante complexa e dependente da dose administrada. Contudo, Briceño et al. (2012) relataram que a administração intravenosa de xilazina causa bradicardia dose-dependente, acompanhada de hipertensão arterial transitória e seguida de hipotensão.

$\mathrm{O}$ uso de agonista $a 2$-adrenérgico irá causar de depressão respiratória secundária a depressão do sistema nervoso central, que é originada pela estimulação de receptores a2-adrenérgicos. Em cabras, foi observada redução da frequência respiratória, sendo esta atribuída à absorção sistêmica da clonidina, causando sedação e depressão central da ventilação (NATALINI; CRUZ; BOPP, 2011). Embora haja relatos de que 22 -adrenoceptores agonistas possam causar bradipneia, neste trabalho, todos os parâmetros respiratórios apresentaram-se estáveis em todos os grupos. Sabe-se que, ao contrário dos anestésicos voláteis, que causam depressão cardiorrespiratória dose-dependente, normalmente a anestesia epidural não produz esses efeitos.

Existem diferentes técnicas de avaliação de analgesia. Neste trabalho, foi utilizada a Escala de Dor da Universidade de Melbourne (University of Melbourne Pain Scale - UMPS) com o objetivo de obter uma acurácia elevada para limitar a subjetividade da avaliação. Mataqueiro et al. (2000), clínicos de campo e, muitas vezes, anestesistas parecem considerar que a sedação profunda acarretaria certo grau de analgesia. Esse conceito, embora contestado em várias publicações científicas, tem sua razão, pois resultados obtidos com romifidina 
e xilazina indicam que, dependendo do método utilizado para se avaliar a dor, pode existir uma confusão entre os efeitos sedativo e analgésico. Essa observação é importante, pois indica que nem sempre que um animal não reage à dor significa que ele não a esteja sentindo, pois é possível que o método utilizado para a avaliação da analgesia não tenha sido adequado, uma vez que a resposta ao estímulo doloroso talvez esteja mascarada pelo efeito sedativo.

\section{Conclusão}

A administração epidural de xilazina ou detomidina nas doses utilizadas em solução fisiológica de $0,9 \%$, até perfazer um volume total de $0,3 \mathrm{ml} / \mathrm{kg}$, produz analgesia intensa durante o período transcirúrgico e pós-operatório, com ausência de depressão respiratória, bradicardia ou hipotensão. (-)

\section{Referências}

ALMEIDA, R. M. et al. Efeitos da administração epidural de amitraz, xilazina ou dimetil sulfóxido em vacas. Arquivo Brasileiro de Medicina Veterinária e Zootecnia, Belo Horizonte, v. 56, n. 6, p. $723-732,2004$.

BRAGA, S. M. Uso de fármacos agonistas dos receptores $\alpha-2$ adrenérgicos em medicina veterinária. 2012. 27 f. Seminário (Seminários Aplicados do Curso de Pós-graduação em Ciência Animal) - Escola de Veterinária, Universidade Federal de Goiás, Goiânia, 2012.

BRICEÑO, E. C. et al. Efectos cardiovasculares de la xilazina en conejos: estudios in vivo e in vitro. Revista de la Facultad de Ciencias Veterinarias, Maracay, v. 53, n. 1, p. 3-12, 2012.

BRONDANI, J. T. et al. Analgesia epidural com clonidina ou romifidina em cães submetidos à cirurgia coxofemoral. Arquivo Brasileiro de Medicina Veterinária e Zootecnia, Belo Horizonte, v. 56, n. 2, p. 175-192, 2004.

CARVALHO, P. S. L. et al. A. Avaliação de alguns parâmetros ecocardiográficos do gato-do-mato (Leopardus tigrinus), mantido em cativeiro e submetido a anestesia com xilazina e quetamina. Arquivo Brasileiro de Medicina Veterinária e Zootecnia, Belo Horizonte, v. 59, n. 3, p. 695-699, 2007.

GASPARINI, S. S. et al. Anestesia epidural com ropivacaína, lidocaína ou associação de lidocaína e xilazina em cães: efeitos cardiorrespiratório e analgésico. Ciência Rural, Santa Maria, v. 37, n. 2 , p. $418-424,2007$

GUIRRO, E. C. B. P. et al. Injeção epidural preventiva de xilazina ou amitraz em equinos: efeitos clínicos e comportamentais. Ciência Rural, Santa Maria, v. 39, n. 2, p. 442-446, 2009.
LAVOR, M. S. L. et al. Estudo comparativo dos efeitos da xilazina, butorfanol, quetamina e lidocaína por via epidural em cães. Ars Veterinaria, Jaboticabal, v. 20, n. 2, p. 195-202, 2004.

MARQUES, J. A.; PEREIRA, D. A.; MARQUES, I. C. S. Associação entre midazolam e detomidina na medicação pré-anestésica para indução da anestesia geral com cetamina em potros. Arquivo Brasileiro de Medicina Veterinária e Zootecnia, Belo Horizonte, v. 61 , n. 6, p. 1290-1296, 2009.

MASSONE. F. Anestesiologia veterinária: farmacologia e técnicas. 6. ed. Rio de Janeiro: Guanabara Koogan, 2011. 428 p.

MATAQUEIRO, M. I. et al. Estudo comparativo dos efeitos sedativos e antinociceptivos da xilazina e romifidina em ratos e camundongos. Ars Veterinaria, Jaboticabal, v. 16, n. 3, p. 165$170,2000$.

NATALINI, C. C; CRUZ, F. S. F; BOPP, S. Analgesia epidural com clonidina ou sufentanil epidural em cadelas submetidas à ovariosalpingohisterectomia sob anestesia geral inalatória. Acta Scientiae Veterinariae, Porto Alegre, v. 39, n. 4, p. 1-9, 2011. Disponível em: <https://goo.gl/Xnp3az>. Acesso em: 15 nov. 2015.

POHL, V. H. Anestesia epidural com alfa2-agonistas e lidocaína para realização de ovariossalpingohisterectomia em cadelas. 2010. 52 f. Dissertação (Mestrado em Medicina Veterinária) Centro de Ciências Rurais, Universidade Federal de Santa Maria, Santa Maria, 2010.

PRADO FILHO, O. R. et al. Xilazina como pré-medicação para anestesia com tiopental sódico em cães. Acta Cirurgica Brasileira, São Paulo, v. 15, n. 2, 2000. Disponível em: <https://goo.gl/ g1LGHC>. Acesso em: 20 nov. 2015.

RIBEIRO, G. et al. Efeitos de detomidina e xilazina intravenosa sobre as variáveis basais e respostas comportamentais em bovinos. Arquivo Brasileiro de Medicina Veterinária e Zootecnia, Belo Horizonte, v. 64, n. 6, p. 1411-1417, 2012.

ROSA, A. C. A farmacocinética e os efeitos sedativos e comportamentais dos cloridratos de xilazina e de detomidina, administrados por diferentes vias, em asininos Nordestinos (Equus asinus). 2014. $117 \mathrm{f}$. Tese (Doutorado em Anestesiologia) - Faculdade de Medicina de Botucatu, Universidade Estadual Paulista, Botucatu, 2014.

SILVA, G. B. Injeção peridural de lidocaína associada à xilazina ou detomidina na prevenção da dor pós-incisional em éguas. 2009. 46 f. Dissertação (Mestrado em Cirurgia Veterinária) - Faculdade de Ciências Agrárias e Veterinárias, Universidade Estadual Paulista, Jaboticabal, 2009.

VALADÃO, C. A. A; DUQUE, J. C; FARIAS, A. Administração epidural de opioides em cães. Ciência Rural, Santa Maria, v. 32, n. 2, p. 347-355, 2002 